\title{
The glueball spectrum at large $\mathbf{N}$
}

\author{
Biagio Lucini \\ School of Physical Sciences, Swansea University \\ Singleton Park, Swansea SA2 8PP, UK \\ E-mail: b.lucini@swansea.ac.uk
}

\section{Antonio Rago}

Department of Physics, Bergische Universität Wuppertal

Gaussstr. 20, D-42119 Wuppertal, Germany

E-mail: rago@physik.uni-wuppertal.de

\section{Enrico Rinaldi*}

SUPA, School of Physics and Astronomy, University of Edinburgh

Edinburgh EH9 3JZ, UK

E-mail: e.rinaldi@sms.ed.ac.uk

The lowest-lying glueball masses are computed in $\mathrm{SU}(N)$ gauge theory on a spacetime lattice for constant value of the lattice spacing $a$ and for $N$ ranging from 3 to 8 . The lattice spacing is fixed using the deconfinement temperature at temporal extension of the lattice $N_{T}=6$. The calculation is conducted employing in each channel a variational ansatz performed on a large basis of operators that includes also torelon and (for the lightest states) scattering trial functions. This basis is constructed using an automatic algorithm that allows us to build operators of any size and shape in any irreducible representation of the cubic group. A good signal is extracted for the ground state and the first excitation in several symmetry channels. It is shown that all the observed states are well described by their large $N$ values, with modest $\mathscr{O}\left(1 / N^{2}\right)$ corrections. In addition spurious states are identified that couple to torelon and scattering operators.

The XXVIII International Symposium on Lattice Field Theory, Lattice2010

June 14-19, 2010

Villasimius, Italy

\footnotetext{
* Speaker.
} 


\section{Introduction}

$\mathrm{SU}(N)$ gauge theories in the large $N$ limit play a central role in the gauge-gravity correspondence and have become the subject of a line of numerical investigations on the lattice. In addition to determining values for observables in the large $N$ limit, lattice calculations provide their corrections at finite $N$. As dictated by the diagrammatic expansion [1], these corrections can be expressed as a power series in $1 / N^{2}$ for the quenched theory and in $1 / N$ in the dynamical case. The emerging picture is that at least for the quenched theory only the leading correction of $\mathscr{O}\left(1 / N^{2}\right)$ is sufficient to describe the system at any finite value of $N$ bigger than two at a level of accuracy of the order of a few percents. In order to assess the reliability of various analytical methods based on the large $N$ framework (which often have to resort to other approximations in addition to taking the large $N$ limit), it is important to compare their predictions to the lattice data for observables that are well under control in both approaches. The glueball spectrum in the pure Yang-Mills theory is one of the easiest observables to compare. Previous numerical calculations at large $N$ have been recently reviewed in [2].

In this work we provide the first determination of the large $N$ glueball spectrum (obtained with an extrapolation including values of $N$ up to eight) in several irreducible representations of the lattice rotational group and for both values of parity and charge conjugation. We are also able to disentangle genuine single-particle states from spurious or multi-particle resonances that are present on finite volume lattice simulations.

\section{The method}

The lattice discretisation of $\mathrm{SU}(N)$ Yang-Mills theory used throughout this work is entirely conventional. We consider the system defined on an isotropic four-dimensional torus of linear size $L$. If $a$ is the lattice spacing, the number of points in each direction is given by $N_{L}=L / a$. We used the Wilson action for the lattice theory, given by

$$
S=\beta \sum_{i, \mu>v}\left(1-\frac{1}{N} \operatorname{Re} \operatorname{Tr}\left(U_{\mu v}(i)\right)\right)
$$

where $U_{\mu v}(i)$ is the parallel transport of the link variables along the elementary lattice plaquette and $\beta$ is defined as $\beta=2 N / g_{0}^{2}$, with $g_{0}$ the bare gauge coupling. In order to compare quantities at fixed lattice spacing across different $\mathrm{SU}(N)$ groups, it proves useful to set the scale using the (pseudo-)critical coupling of the deconfinement transition at fixed temporal extent $N_{T}=6$. A $N_{L}=12$ lattice for $\beta=\beta_{c}\left(N_{T}=6\right)$ gives a glueball spectrum in the scaling region and free from large finite size artefacts [3].

In general, masses of bound states on the lattice are extracted from the exponential decay of connected correlation functions between operators with the desired quantum numbers. In lattice YangMills theory these operators are constructed using traces of path ordered products of links around closed loops. Moreover, the links used in the operators are smeared and blocked [4,5] several times in order to obtain smooth operators on physical length scales that project onto the low-lying states of the spectrum. A variational ansatz for the correlators is also employed: for every set of quantum numbers $J^{P C}$ we measure a matrix of correlators between different operators and we look for their 
linear combination that has the best overlap onto the state we are interested in. This allows us to obtain the mass of the groundstate and of the first excitations of the spectrum with the smallest possible systematic errors (for more details on the variational technique see Ref. [6]).

At finite volume, the single-particle glueball spectrum receives non-negligible corrections from multi-glueball states. Moreover, when the system is closed with periodic boundary conditions (like in our case) topological excitations wrapping the compact direction (torelons) with the same quantum numbers of glueballs appear; if not correctly accounted for, these states can affect significantly the measured glueball spectrum. In order to control these spurious contributions, we include in the variational set operators that best overlap with two-glueball and torelon states.

\section{The operators}

On the lattice, the continuum quantum numbers $J^{P C}$ are replaced by the ones labelling the irreps. of the cubic symmetry group combined with reflections and charge conjugation, giving a total of 20 symmetry channels $R^{P C}$. An operator in the channel $R^{P C}$ is obtained from the gaugeinvariant, vacuum-subtracted operator $\overline{\mathscr{O}}(t)$ by means of

$$
\Phi(t)=\sum_{i} c_{i} \mathscr{R}_{i}(\overline{\mathscr{O}}(t))
$$

In the equation above, $\mathscr{R}_{i}$ represents a transformation belonging to the full symmetry group of the system and the coefficients $c_{i}$ depend on the channel $R^{P C}$ [7].

We built three different classes of operators, one that mainly projects on single-glueball states, one for two-glueball scattering states and one for torelon excitations. The single-trace operator that we use to project onto glueball states is simply defined as

$$
\mathscr{O}_{G}(t)=\frac{1}{N_{L}^{3}} \sum_{\vec{x}} \operatorname{Tr} \prod_{l \in \mathscr{C}(\vec{x})} U_{l}
$$

In our definition of the variational set we used a wide range of different closed loops $\mathscr{C}$, with lengths ranging from 4 to 8 lattice spacing. In Fig. 1 we summarize the closed loops used in our simulations and the number of operators built in each channel. Each of these operators is then smeared and blocked four times.

An operator that projects onto scattering states of two glueballs is a double-trace operator. Our trial operators for scattering states have the form

$$
\mathscr{O}_{S}(t)=\left(\mathscr{O}_{G}(t)-\left\langle\mathscr{O}_{G}\right\rangle\right)^{2},
$$

where we used the definition of the single-trace operator in Eq. (3.2) and the same shapes listed in Fig. 1. The local subtraction of $\left\langle\mathscr{O}_{G}\right\rangle$ is crucial in order to obtain the correct two-point function. A more detailed discussion about the scattering operators can be found in Ref. [6].

The torelon operators have been created from products of two Polyakov loops $l_{v}$ winding around opposite directions, in order to obtain an operator which transforms trivially under the centre of the gauge group. We defined our operators as

$$
\mathscr{O}_{T}(t)=\frac{1}{2 N_{L}^{2}} \sum_{\mu \neq v} \sum_{x} l_{v}(x, t) l_{v}^{\dagger}(x+\hat{\mu} a, t)
$$


where the sum over $\mu$ runs on the spatial directions orthogonal to the one of the loops. By choosing different shapes for the combination $l_{v}(x, t) l_{v}^{\dagger}(x+\hat{\mu} a, t)$, we can obtain a fairly large variational set projecting on torelon states as summarized in Fig. 2.

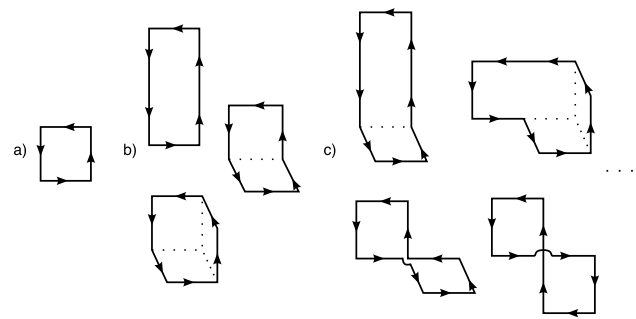

\begin{tabular}{c|cccc} 
& ++ & -+ & +- & -- \\
\hline$A_{1}$ & 8 & 2 & 1 & 3 \\
$A_{2}$ & 3 & 1 & 3 & 3 \\
$E$ & 22 & 7 & 7 & 14 \\
$T_{1}$ & 19 & 24 & 48 & 27 \\
$T_{2}$ & 44 & 33 & 33 & 29
\end{tabular}

Figure 1: (Left) Shapes of basic prototypical paths used to construct operators $\mathscr{O}_{G}(t)$. (Right) Number of single-glueball operators included in the variational set for each symmetry channel. Each operator is then smeared 4 times.
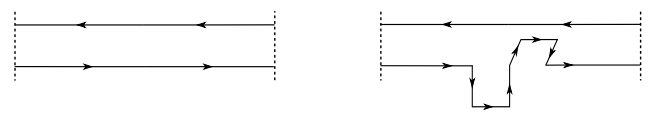

\begin{tabular}{c|cccc} 
& ++ & -+ & +- & -- \\
\hline$A_{1}$ & 2 & 1 & 0 & 0 \\
$A_{2}$ & 1 & 0 & 1 & 1 \\
$E$ & 7 & 3 & 3 & 3 \\
$T_{1}$ & 3 & 3 & 14 & 9 \\
$T_{2}$ & 9 & 9 & 8 & 3
\end{tabular}

Figure 2: (Left) Paths used for the construction of operators $\mathscr{O}_{S}(t)$ coupling with torelon states. Periodic Boundary Conditions apply at the edges represented by the dashed lines. (Right) Number of $\mathscr{O}_{S}(t)$ operators included in the variational set for each symmetry channel. Each operator is then smeared 4 times.

\section{The results}

The states obtained after the variational procedure can be decomposed into their projection onto the pure glueball states, the scattering states and onto the torelons:

$$
\hat{\Phi}=\sum_{i} v_{i} \Phi_{i}(t) \equiv \alpha_{G} \Phi_{G}+\alpha_{S} \Phi_{S}+\alpha_{T} \Phi_{T} ; \quad \operatorname{mix}_{A}=\frac{\left|\alpha_{A}\right|^{2}}{\sum_{i}\left|\alpha_{i}\right|^{2}} ; \quad A \in\{G, S, T\},
$$

where we also defined the relative projection $\left(\operatorname{mix}_{A}\right)$. Masses extracted from correlators of $\hat{\Phi}$ with $\operatorname{mix}_{S}, \operatorname{mix}_{T} \geq 20 \%$ can not be reliably interpreted as pure single-glueball resonances, because spurious states are expected to propagate between the two sources.

After performing the variational calculation, the diagonal elements of the correlation matrix are fitted with the single-cosh ansatz, which assumes that only one state dominates the signal. We are often able to obtain overlaps of the order of 0.95 , which proves the validity of the original variational ansatz. As a consequence, the fit generally works very well on the range $1 \leq t \leq 4$ [6].

For all gauge groups, there is a high mixing between narrow glueball trial states and torelon states in the first excitation of the $E^{++}$and in the second excitation of the $A_{1}^{++}$. Other states with a consistent mixing with the torelons are the $T_{2}^{+-}$and the $T_{1}^{--}$, the latter mostly for $N=3,4$. Since a calculation involving scattering states is much more demanding in terms of computer time, we use the results 
from the computation involving only single-particle and torelon operators to target the channels where mixing with multi-particle states is expected to affect significantly the results. At large $N$, this is expected to happen for the excited states that are close to twice the energy of the groundstate. It is then clear that the channel in which scattering states can potentially influence the measured spectrum in a relevant way is the $A_{1}^{++}$, where we can extract several excitations. We perform calculations on separate sets of operators in the $A_{1}$ channel (the full set and the sets obtained excluding in turn scattering, torelon and single-glueball operators). The remarkable property shown by this calculation is that when only scattering and torelon operators are used the lowest-lying state has a mass that is much higher (roughly by a factor of two) than the mass of the groundstate extracted with the full variational basis. Moreover, the latter appears always when single-particle operators are included in the calculation. This is an indication that our multi-glueball set of operators projects only on scattering states, as it should be. The scattering state seems to be slightly above the first excited single-glueball excitation at any value of $N$ (see for example the SU(3) spectrum of the $A_{1}^{++}$channel in Fig. 3, where two different volumes are investigated).
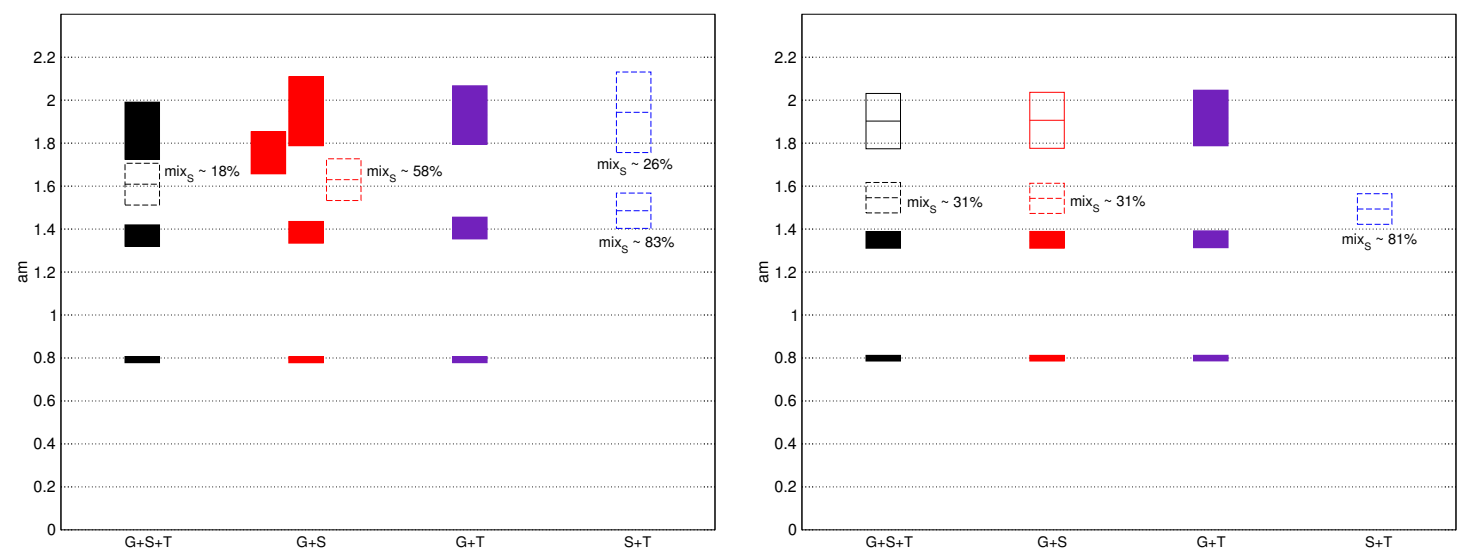

Figure 3: Variational calculation for $\mathrm{SU}(3)$ using different sets of operators on a $N_{L}=12$ lattice (left) and a $N_{L}=18$ lattice (right). The unfilled symbols represent masses that cannot be reliably interpreted as pure glueballs.

Having determined the single-glueball spectrum at different values of $N$ from 3 to 8 , we extrapolate to the $N=\infty$ limit using the functional form

$$
\operatorname{am}_{G}(N)=\operatorname{am}_{G}(\infty)+c / N^{2},
$$

dictated by the diagrammatic expansion and already used with success in Ref. [3]. We find that this ansatz works for all the measured states (including the excitations) for $N \geq 3$. In general, the central value of $c$ is found to be small (always of order one or below), as it is expected for a generic coefficient in a well-behaved expansion. For most of the states we find only modest corrections to the $N=\infty$ value of the mass: with a few exceptions, $c$ is compatible with zero and a fit with only the leading term $\operatorname{am}_{G}(\infty)$ in Eq. (4.2) gives a result that is compatible with the fit that includes also the $\mathscr{O}\left(1 / N^{2}\right)$ correction [6]. In Fig. 4 we show the large- $N$ extrapolation of the groundstate and the first two excitations of the would-be continuum scalar $0^{++}$glueball, together with the groundstate and the first excited state of the tensor $2^{++}$glueballs. 

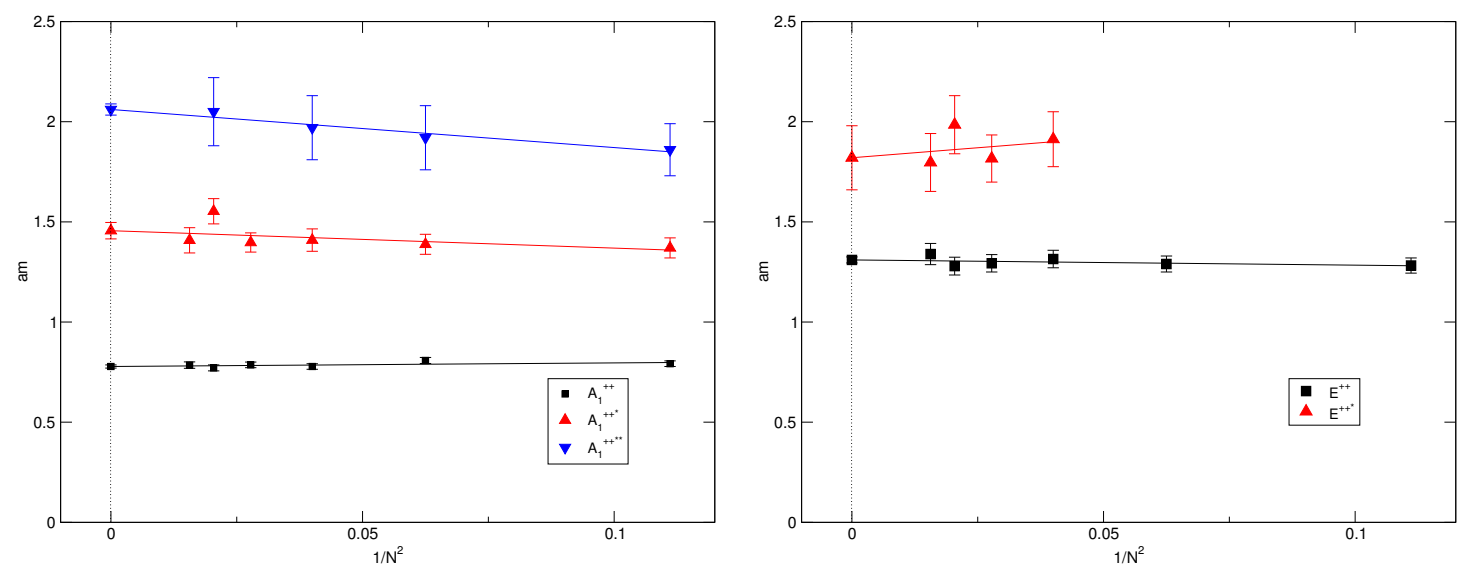

Figure 4: Extrapolation to $N \rightarrow \infty$ of the states in the $A_{1}^{++}$channel (left) and in the $E^{++}$channel (right).

The single-glueball spectrum determined in this work is plotted in Fig. 5 and it is compared with the known spectrum at the same lattice spacing taken from Ref. [3]. The latter work achieves a comparable precision for the $A_{1}^{++}$, the $E^{++}$and the $A_{1}^{++\star}$, but in this study we are able to measure seventeen more states. Moreover, the states present in both studies are compatible.

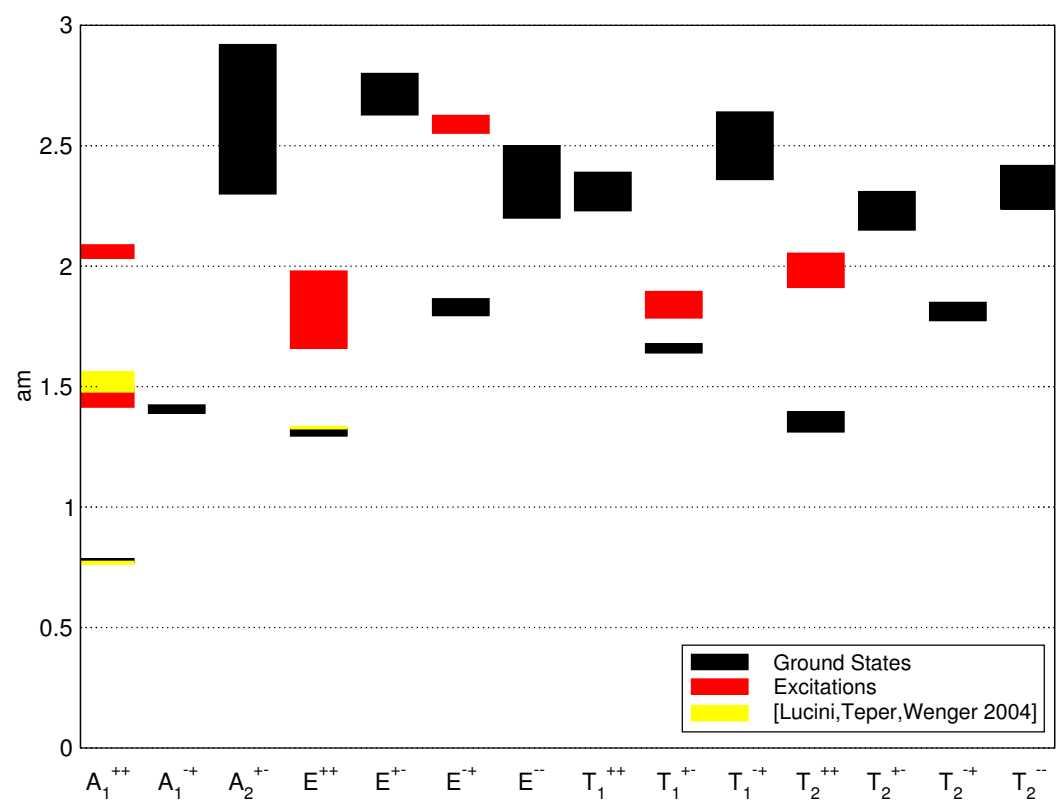

Figure 5: The spectrum at $N=\infty$. The yellow boxes represent the large $N$ extrapolation of masses obtained in Ref. [3].

\section{Conclusions}

In this work, we have studied numerically on the lattice the glueball spectrum in Yang-Mills $\mathrm{SU}(N)$ gauge theories in the large $N$ limit. Using an automated technique for constructing trial wave functionals in all possible symmetry channels, we have built a large variational basis that has 
enabled us to obtain a large number of states, including some excitations. Moreover, the inclusion of functionals that best overlap with scattering and torelon states has allowed us to unambiguously exclude multi-particle states or finite-size artefacts from the spectrum of narrow resonances. This is a significant advance in our understanding of the large $N$ glueball spectrum from first principles. With little or no modification, the technique we have presented in this work will also prove helpful in related problems, like the lattice study of glueballs in QCD and the study of the low-energy spectrum of confining flux tubes.

\section{Acknowledgments}

We thank M. Peardon and M. Teper for discussions on the identification of scattering states and on the construction of scattering operators. Discussions with C. McNeile, H. Meyer, C. Núnez and A. Patella on various aspects of this work are also gratefully acknowledged. Numerical simulations have been performed on a 120 core Beowulf cluster partially funded by the Royal Society and STFC, and on a 100 core cluster at Wuppertal University. The work of B.L. is supported by the Royal Society through the University Research Fellowship scheme and by STFC under contract ST/G000506/1. A.R. thanks the Deutsche Forschungsgemeinschaft for financial support. E.R. is supported by a SUPA Prize Studentship. E.R. acknowledges financial support by the Royal Society in the early stage of this work.

\section{References}

[1] G. 't Hooft, A planar diagram theory for strong interactions, Nucl. Phys. B72 (1974) 461.

[2] M. Teper, Large N, POS LATTICE2008 (2008) 022, [arXiv:0812.0085].

[3] B. Lucini, M. Teper, and U. Wenger, Glueballs and k-strings in $S U(N)$ gauge theories: Calculations with improved operators, JHEP 06 (2004) 012, [hep-lat/ 04040 08].

[4] APE Collaboration, M. Albanese et al., Glueball Masses and String Tension in Lattice QCD, Phys. Lett. B192 (1987) 163-169.

[5] M. Teper, An Improved Method for Lattice Glueball Calculations, Phys. Lett. B183 (1987) 345.

[6] B. Lucini, A. Rago, E. Rinaldi, Glueball masses in the large N limit, JHEP 1008 (2010) 119. [arXiv:1007.3879]

[7] C. Michael and M. Teper, The Glueball Spectrum in SU(3), Nucl. Phys. B314 (1989) 347. 